\title{
JOHANN CASPAR LAVATER IN ESTLAND. ÜBER SEINEN EINFLUSS, EINIGE POR- TRAITS UND EINIGE GEDANKEN ÜBER DIE PORTRAITKUNST
}

\section{LAVATERS BEKANNTHEIT}

Jeder Forscher der Aufklärungszeit und auch der Interessierte weiß, wer Johann Caspar Lavater (1741-1801) war. Die Bekanntheit des in Zürich geborenen und dort lebenden Lavater stützte sich einerseits auf seine außerordentlich charismatische Persönlichkeit sowie auf seine Arbeit als Pfarrer, auf das Verfassen von Liedertexten und auf sein Sammeln von bildnerischer Kunst. Andererseits bescherten Lavater seine analytisch-subjektiven Schriften Berühmtheit, ebenso wie jene auf den Gebieten der Theologie und der Physiognomie, so vor allem die vierbändigen prachtvollen Folianten der „Physiognomische Fragmente, zur Beförderung der Menschenkenntniß und Menschenliebe" (1775-1778). ${ }^{1}$ Im deutschen Sprachraum in der zweiten Hälfte des 18. Jahrhunderts gehörten fast alle großen Persönlichkeiten zum Bekanntenkreis von Lavater. Mehrere von ihnen, wie beispielsweise Johann Wolfgang von Goethe (1749-1832), ${ }^{2}$ waren an der Herausgabe der „Physiognomische Fragmente" als Mitarbeiter tätig.

DOI: http://dx.doi.org/10.12697/BJAH.2016.12.02

Übersetzung aus dem Estnischen von Marju und Olaf Mertelsmann.

Der Aufsatz wurde als Ergebnis eines Stipendiums der Vereinigung der Kunstwissenschaftler und der Kuratoren Estlands (Eesti Kunstiteadlaste ja Kuraatorite Ühing) und eines Forschungsaufenthalts in Zürich verfasst. Ich danke auch Dr. Ursula Caflisch-Schnetzler, Kuratorin der Sammlung Johann Caspar Lavater, für die kritische Lektüre, Hilfe und Unterstützung.

1 Johann Caspar Lavater, Physiognomische Fragmente, zur Beförderung der Menschenkenntniß und Menschenliebe, I-IV Bände (Leipzig, Winterthur: Bey Weidmanns Erben und Reich, und Heinrich Steiner und Compagnie, 1775-1778).

2 Lavater, Physiognomische Fragmente, III, 318. 
Lavaters Ideen, ebenso wie jene der anderen deutschen Aufklärer im Zeitalter des Sturm und Drang (etwa von 1770 bis 1785), verbreiteten sich sehr und nicht nur in Europa, sondern auch in Amerika und in Russland. Viele in der Zeit von Kaiserin Katharina II. (1729-1796) nach Russland gereisten Schweizer ${ }^{3}$ verfügten über Kontakte sowohl zu Gelehrten in St. Petersburg und Moskau als auch zum Zarenhof, so auch Lavater Von der Zarin Katharina II war er so begeistert, dass er ihr ein eigenes Kapitel in den „Physiognomischen Fragmenten” widmete. ${ }^{4}$ Besonders eng und dauerhaft gestalteten sich Lavaters Beziehungen in Russland zu Großfürst Paul (Pavel, 1754-1801) und zu seiner zweiten Gattin, Maria Feodorowna (1759-1828). ${ }^{5}$ Jahre später, als Paul bereits die Zarenkrone trug, gab er dem Oberbefehlshaber der Truppen, die Zürich von den Einheiten Napoleons befreien sollten, Aleksandr Rimskij-Korsakov (17531840), den Befehl mit, dass sobald russische Truppen die Schweiz besetzt hätten, sollte er den „,bekannten Lavater” unter seinen Schutz stellen und bei Lavater nachfragen, ob dieser einen Ehrentitel vom Zarenhof, einen Orden, eine Pension oder etwas anderes erhalten möchte. ${ }^{6}$

Verständlicherweise war Lavater auch unter der deutschbaltischen Elite der Ostseeprovinzen des Russischen Reichs bekannt. ${ }^{7}$ Unter den örtlichen Deutschen verfügten der im heutigen Lettland aktive Theologe

3 Schweizer spielten eine wichtige Rolle dabei, das Russland in der Zeit von Katharina II. europäischer zu machen. In der Russischen Akademie der Wissenschaften gab es in der zweiten Hälfte des 18 ahrhundert 35 schweizerische Mitglieder, darunter die Brüder Bernoulli, Jakob Hermann, Leonhar Euler sowie den zu dem engeren Freundeskreis von Lavater zählenden Georg Gessner. - Edmund Heier, „Das Lavaterbild im geistigen Leben Russlands des 18. Jahrhunderts”, Kirche im Osten, Ban 20 (1977) (Göttingen, 1997), 108-109.

Lavater, Physiognomische Fragmente, III, 323.

Marianna Butenschön, Maria, Kaiserin von Russland. Die Württembergerin auf dem Zarenthron (Darmstadt: Konrad Theiss Verlag, 2015), 115-118, 199-200. S. auch: Lavaters Sammlun Physiognomischer Original Zeichnungen: aus dem Besitz der Zarin Maria Feodorowna. Siehe: http:// retro.seals.ch (zuletzt besucht am 19.01.2016).

Für all das sollte der Oberbefehlshaber Lavater das Schreiben von Zar Paul I. übergeben, in dem er an seine Reise in die Schweiz und seine Bekanntschaft mit Lavater erinnerte. Es eröffnete ihm Anerkennung als einem großen Humanisten und ein Bedauern darüber, dass er der Franzosischen Revolution zum Opfer gefallen war. Es beschrieb die an Rimskij-Korsakov übergebene Aufgabe, fü Lavater zu sorgen, und wünschte ihm den Schutz der Heiligen. Ob der von Paul I. am 23. Septembe 1799 in Gatčina unweit von St. Petersburg verfasste Brief Lavater je erreichte, ist nicht bekannt. - Heie „Das Lavaterbild im geistigen Leben Russlands des 18. Jahrhunderts", 116-117

Siehe: Kulturaustausch. Baltisches Echo auf Gelehrte in der Schweiz und in Deutschland, hrsg. von Hanspeter Marti (Kön, Weimar, Wien: Bohlau Verlag, 2014). Im Estland waren von Lavater theologischen Schriften beispielsweise die folgenden erhaltlich: Handbibel für Leidende (Basel: Mülle 1830), Zwo Predigten gehalten zu Ems (Frankfurt am Main: Eichenbergische Erben, 1774), Aussichten in die Ewigkeit (Zurich: Bey Orell, Gessner und Compagnie, 1769-1771, 1781) und Schreiben an seine
Freunde (Winterthur: Heinrich Steiner und Compagni, 1776). und vielseitige Förderer des lokalen kulturellen Lebens, Liborius von Bergmann (1754-1823), ${ }^{8}$ sowie der Rigenser Verleger Johann Friedrich Hartknoch (1740-1789), der den Buchmarkt des Baltikums mit demjenigen Europas verbunden hatte, über Kontakte mit Lavater. ${ }^{9}$ Von Lavater und von seiner Lehre der Physiognomie war die in Kurland aktive Poetin und Schriftstellerin Elisabeth von der Recke (1754-1833) beeindruckt. ${ }^{10}$ Der Herzog von Kurland und Semgallen, Peter von Biron (1724-1800), nutzte die Dienste Lavaters und seines Lehrers Johann Jakob Bodmer (1698-1783) bei der Suche von Lehrkräften für die in Mitau (heute Jelgava) gegründete Academia Petrina. ${ }^{11}$ Der Professor für Philosophie, Gottlob David Hartmann (1752-1775), befand sich bis zu seinem Tode im Briefverkehr sowohl mit Lavater als auch mit Bodmer. Sein Portrait nutzte Lavater als Bildquelle in seinen physiognomischen Studien, genauer gesagt - als Beispiel für die Gesichtszüge eines jungen Genies (im Profil). ${ }^{12}$

Von den auf dem estnischen Gebiet aktiven deutschen Aufklärern kannten Lavater, wenn nicht persönlich (was sich wegen des Fehlens von Quellen auch nicht ausschließen lässt), so doch zumindest dessen Werke der Pastor der Kirche von Oberpahlen (estn. Põltsamaa) und Ecks (estn. Äksi), August Wilhelm Hupel (1737-1819) ${ }^{13}$, sowie der Professor der Kaiserlichen Universität zu Dorpat (estn. Tartu), Johann Simon Karl Morgenstern (1770-1852). ${ }^{14}$ Dasselbe kann über einen Begründer der Künstler-Dynastie der von Kügelgens, Franz Gerhard von Külgelgen

8 Juta Keevallik, „Märkmeid Anton Graffi portreedest Eestis ja Lätis”, Töid kunstiteaduse ja -kritika alalt 2 (Tallinn: Kunst, 1978), 335; Klaus Garber, Schatzhäuser des Geistes. Alte Bibliotheken un Büchersammlungen im Baltikum (Köln, Weimar, Wien: Böhlau Verlag, 2007), 107.

9 Johann Caspar Lavater Ausgewählte Werke. Ergänzungsband: Verzeichnisse der Korrespondenz und des Nachlasses in der Zentralbibliothek Zürich, hrsg. von Christoph Eggenberger und Marlis Stähli (Zürich: NZZ Verlag, 2007), 79, 186; Heinz Ischreyt, „Zur Rezeption Lavaters in Kurland und Livland", Nordost-Archiv. Zeitschrift für Regionalgeschichte und Landeskunde 17, 73 (1984), 53-68. 10 Johann Caspar Lavater Ausgewählte Werke. Ergänzungsband: Verzeichnisse der Korresponden und des Nachlasses in der Zentralbibliothek Zürich, 117, 226; Heier, „Das Lavaterbild im geistigen
Leben Russlands des 18. Jahrhundert”, 124 .

1 Ursula Caflisch-Schnetzler, „Kommunikation mit dem Baltikum im 18. Jahrhundert”, Noli me nolle. Sammlung Johann Caspar Lavater, Jahresschrift (Zürich, 2015), 8

12 Ursula Caflisch-Schnetzler, „Pädagogik und Kommunikation - Zürich und das Baltikum”, Kulturaustausch. Baltisches Echo auf Gelehrte in der Schweiz und in Deutschland, 146, 174, 179-181. G. D. Hartmanns Portrait: Lavater, Physiognomische Fragmente, I, 258.

13 In der Lesegesellschaft von Oberpahlen bestand für den Leser die Möglichkeit, sich mit Lavaters "Physiognomischen Fragmenten” bekannt zu machen. Indrek Jürjo. Liivimaa valgustaja August Wilhelm Hupel 1737-1819 (Tallinn: Rigiarhiiv, 2004), 131

14 Briefe an Kant, von Garve, Hamann, Kästner, Lavater, Lichtenberg, Moses Mendelssohn, Selle, Sulzer, Wieland und Wyttenbach. - Dörptische Beyträge für Freunde der Philosophie, Litteratur Herausgebers gedruckt bey J. C. Schünmann (Leipzig: in Commission bey P. G. Kummer, 1817), 87-124. 
(1772-1820), und seine Gattin gesagt werden. Helene Marie von Kügelgen (1774-1842) hatte Lavaters „Physiognomische Fragmente” gelesen, ${ }^{15}$ Gerhard von Kügelgen ließ sich von ihnen schließlich gar beeinflussen. Das letztere bestätigt eine ihm und seinem Zwillingsbruder, dem Künstler Karl Ferdinand von Kügelgen (1772-1832), gewidmete, neue Untersuchung. ${ }^{16}$

Erwähnt werden sollten auch die Zusammentreffen des aus Livland stammenden jungen Künstlers und Schriftstellers Karl Gotthard Grass (1767-1814) und des Hauslehrers, zukünftigen Architekten der Universität Dorpat und ihres Professors, Johann Wilhelm Krause (1757-1828), mit Lavater in der Schweiz im Jahre 1796/1797. Für sie galt wie für andere, für die auf der Durchreise nach Italien in Zürich einen Halt machenden gebildeten deutschen Männer war es eine Ehrensache, sich mit Lavater zu treffen und mit ihm Bekanntschaft zu machen"17.

Besonders hervorgehoben zu werden verdient aber in diesem Beitrag, der sich auf Lavaters Portraits und auf seine Gedanken zur Portraitkunst konzentriert, dessen Bekanntschaft mit dem Ratsapotheker von Reval (estn. Tallinn), Johann Burchard VII. (Burchart, 1748-1808), dem Vater des Gründers von Mon Faible (des ersten Museums der Stadt), Johann Burchard VIII. (1776-1838). In der Burchard-Sammlung des Estnischen Geschichtsmuseums (estn. Eesti Ajaloomuuseum) ist das erste Portrait Lavaters in Estland erhalten, das offenbar von diesem selbst nach Reval gesandt worden war. Die Tatsache, dass Burchard mit Lavater wenigs-

15 Helene Marie von Kügelgen (geb. Zoege von Manteuffel), Sächsische Biografie: http://saebi.isgv. de/biografie/Lilla_von_K\%C3\%BCgelgen_(1774-1842) (zuletzt besucht am 22.09.2015).

16 Dresdeni ja Peterburi vahel. Kunstnikest kaksikvennad von Kügelgenid. / Between Dresden and St. Petersburg. Artist Twin Brothers von Kügelgen, hrsg. von Kadi Polli (Tallinn: Eesti Kunstimuuseum - Kadrioru Kunstimuuseum, 2013), 48, 106. In Beziehung auf die ortlichen Kunstler kann noch erganzt werden, dass es möglich ist, dass das heutzutage der Estnischen Nationalbibliothek (estn. Eesti Rahvusraamatukogu) gehörende Exemplar der „Physiognomischen Fragmente” einst im Besitz vo Constantin von Ungern-Sternberg war. Ich danke Juta Keevallik für diesen Hinweis

17 Zusätzlich zum Zitat: Unter den Lavater besuchenden Deutschbalten befand sich auch der au Livland stammende Schriftsteller Jacob Michael Reinhold Lenz, der sich im Jahr 1777 in der Schweiz aufhielt. 1778 besuchte Lavater den oben erwähnten Pastor Liborius Bergmann, der mit Goethe und Lavaters jungerem Bruder Diethelm zur selben Zeit an der Universität Leipzig studiert hatte. - Arvo Tering, Eesti-, livi- ja kuramaalased Euroopa ulikoolides 1561-1798 (Tartu: Kirjastus Eest Wjalooarhiv, 2008), 484-485, Kadi Polli, „, Eelromantism Johann Winhelm Krause loomingus, , Johan Eesti kunsti lugu (Tallinn: Varrak, 2007), 450. tens für zehn Jahre (1779-1789) im Briefwechsel stand ${ }^{18}$ sollte einen nicht verwundern - Lavaters persönliche Korrespondenz war nämlich außergewöhnlich groß. Alleine in der Züricher Zentralbibliothek sind mehr als 21.400 an ihn adressierte oder von ihm verfasste Briefe erhalten. ${ }^{19} \mathrm{Zu}$ diesen kommen noch die beim Adressaten verbliebenen Schreiben, darunter auch die im Estnischen Geschichtsmuseum und in der Universitätsbibliothek in Tartu (estn. Tartu Ülikooli Raamatukogu) erhaltenen und erst auf eine Bearbeitung wartenden Briefe und Notizen. ${ }^{20}$

\section{LAVATERS PORTRAITS IN ESTLAND}

Zusätzlich zu den von Lavater verfassten Werken und seinen Briefen finden sich in Estland mehrere Portraits des bekannten Schweizers. Das Werk, das zur Burchard-Sammlung der kulturhistorischen Sammlung des Estnischen Geschichtsmuseums gehört, ist eins von vier hier bekannten Portraits Lavaters (als Einzelobjekt, nicht als Buchillustration). Es handelt sich um ein Miniaturgemälde in rundem Format, welches die Initialen J. C. L. und das Datum 30.VI.1792 trägt (Abb. 1). ${ }^{21}$ Diese Datierung belegt, dass Lavaters Freundschaft mit Burchard zeitlich den erhaltenen Briefwechsel überstieg und mindestens dreizehn Jahre lang andauerte.

Dank eines in Zürich erhaltenen Briefes Burchards ist aber bekannt, dass das im Estnischen Geschichtsmuseum aufbewahrte Miniaturgemälde nicht das einzige Bildnis ist, welches Lavater von sich an seinen Revaler Freund sandte. Das erste Bildnis, verschickt im Jahr 1779 oder etwas

18 Johann Caspar Lavater Ausgewählte Werke. Ergänzungsband: Verzeichnisse der Korrespondenz und des Nachlasses in der Zentralbibliothek Zürich, 59, 165; Der älteste bekannte Brief von Lavate an Burchard stammt aus dem Jahr 1779 (Archiv des Estnischen Geschichtsmuseums, f. 114, n. 1, 4), das letzte Schreiben von Burchard an Lavater aber aus dem Jahr 1789 (Zentralbibliothek Zürich, FA_Lav_Ms_504_243-249, eine digitale Kopie der Dokumente befindet sich im Besitz der Verfasserin
dieses Aufsatzes). dieses Aufsatzes).

19 Johann Caspar Lavater Ausgewählte Werke. Ergänzungsband: Verzeichnisse der Korrespondenz und des Nachlasses in der Zentralbibliothek Zürich, 9

20 Archiv des Estnischen Geschichtsmuseums: f. 114, n. 1, s. 4, 9-23 (J. C. Lavaters Schreiben an . Burchard mitsamt Unterschrift); Lavaters kurze Notizen, 1793, 1796 - Bibliothek der Universität Tartu 1658 a, b, c.

21 Inventarnummer des Gemäldes; EAM AM 6077, K 270. Enkaustik (2), verglast, 7,6x71 cm. Das Portrait ist in Johann Burchards VIII. Familiensammlung im Jahr 1822 begonnenen Katalog (in einer erhaltenen Abschrift aus dem Jahre 1830) unter der Rubrik „Kunst-Arbeiten alter und neuer Zeit unter der Nummer 14 mit folgenden Worten verortet: Das Bild Lavater's in Wacks-Mahlerey von ihm

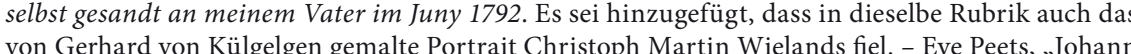
von Gerhard von Kulgelgen gemalte Portrait Christoph Martin Wielands fiel. - Eve Peets, „Johan Burchardi kollektsioon Eesti Ajaloomuuseumis", Töid ajaloo alalt 4 (Tallinn: Eesti Ajaloomuuseum,
2002, 121, 124 . 


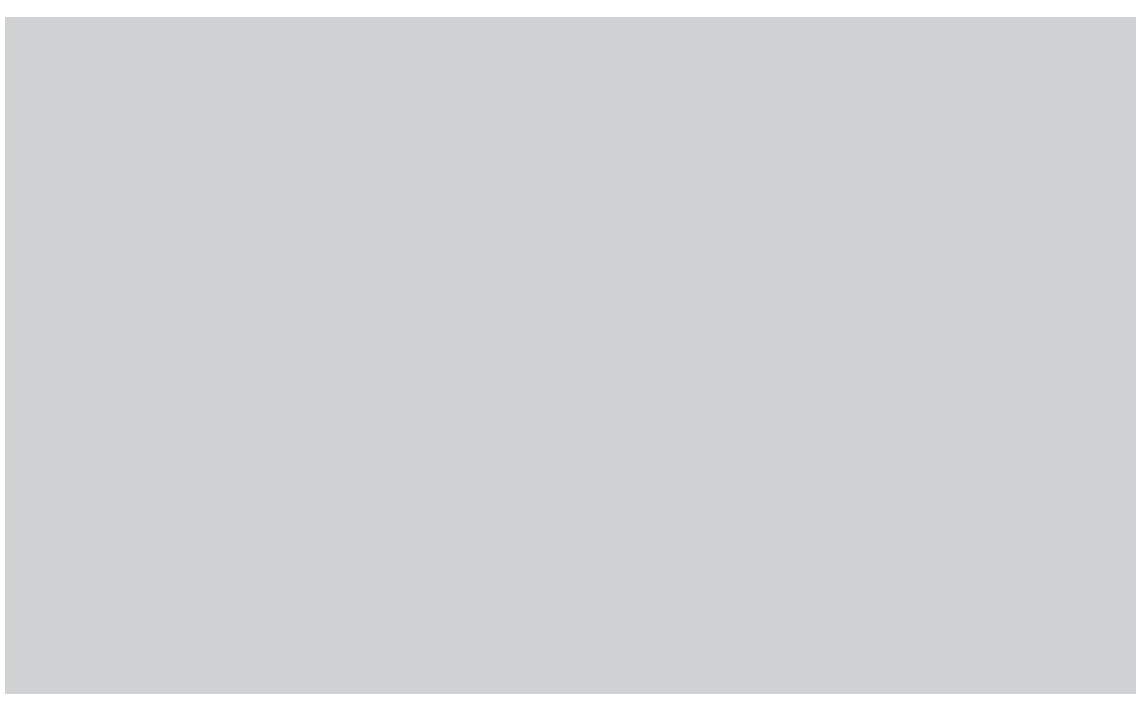
Abb. 1. Miniaturportait Johann Caspar Lavaters, Enkaustik, 1792. Estnisches Geschichtsmuseum.
Abb. 2. Johann Heinrich Lips, Portrait Johann Caspar Lavaters. Foto: Wikimedia Commons.

früher, ${ }^{22}$ war ein Silhouettenportrait bzw. ein Schattenriss. ${ }^{23}$ Dies ist bedeutungsreich in Anbetracht der Tatsache von Lavaters Forschungen zur Physiognomie und seinen Einschätzungen - das Profil sagt am meisten über das Wesen und seine geistigen Fähigkeiten eines Menschen aus. Umso mehr zählt, dass bekannt ist, dass für Lavater ein Schattenriss über keinen ästhetischen Wert verfügte, sondern nur von wissenschaftlicher und analytischer Bedeutung war. ${ }^{24}$

Weiterhin ist dank der erwähnten Briefe auch bekannt, mit welchen Emotionen das Portrait Lavaters in Reval aufgenommen wurde. Das Schreiben beginnt mit der Wendung Verehrungswürdiger Menschenfreund und setzt sich fort in dem für diese Epoche typischen, überschwänglichen Stil mit dem Dank für den Schattenriss. Aus demselben Brief ergibt sich auch, dass die beiden über Lavaters Portraitbildnis gesprochen hatten, welches aber noch fertigzustellen sei und dessen versprochene Zeichnung Burchard für sich haben möchte - denn alles, was aus Lavaters

22 Leider ist der Brief undatiert, doch er ging dem folgenden archivierten Schreiben Burchards, das Im Juli 1779 eintraf, voraus. - Zentralbibliothek Zürich, FA Lav. Ms 504.243, 1¹/4.

23 Karin Althaus, „Die Physiognomik ist ein neues Auge.” Zum Porträt in der Sammlung Lavater (Basel Universität Basel, 2003), 153. Die Dissertation handelt es sich um die physiognomische Sammlung welche sich he
24 Ibidem.
Händen zu ihm gelange, besitze einen unendlich großen Wert. ${ }^{25}$ Von welchem Portrait Lavaters exakt die Rede war, kann aus dem Brief heraus nicht erkannt werden, ebenso bleibt die Frage ungeklärt, wo der nach Reval geschickte Schattenriss verblieben ist. In dem Verzeichnis der Gegenstände von Johann Burchards VIII. Mon Faible ist dieser nicht mehr zu finden, und der Teil der Sammlung, der nach Burchards Tod an das Estländische Provinzialmuseum (und danach an das Estnische Geschichtsmuseums) übergeben wurde, enthielt diesen ebenfalls nicht. ${ }^{2}$

Leider bleibt auch die Frage ohne Antwort, wer der Maler der im Jahr 1792 (anstelle einer Zeichnung?) an Burchard gesandten Miniaturgemäldes Lavaters war? Am meisten ähnelt diese Miniatur einem grafischen Portrait (Abb. 2), dessen Urheber Johann Heinrich Lips (1758-1817) war. Er portraitierte Lavater wiederholt und war dessen produktivster Mitarbeiter. ${ }^{27}$ Die Tatsache, dass Lavater auf diesen Bildern in verschiedene Richtungen schaut, unterstützt die Vermutung dass zwischen beiden Bildern eine Verbindung besteht. Oftmals waren die nach einem Gemälde angefertigten Gravuren spiegelbildlich. Doch ebenso kann vermutet werden, dass das Miniaturgemälde in Burchards Sammlung das Original von Lips ist, welches der Künstler später als Grafik reproduzierte: Lips, der sich auch mit Malerei beschäftigte, war in der Lage, von Lavater ein Miniaturportrait anzufertigen. Ohne ergänzende (technische) Untersuchungen kann ihm dieses Werk jedoch nicht zugeschrieben werden.

Wichtig zu bemerken ist, dass die Portraits in der Universitätsbibliothek in Tartu und im Tallinner Stadtmuseum (estn. Tallinna Linnamuuseum) (Abb. 3, 4) dem Miniaturgemälde und den erwähnten Gravuren von Lips ähneln. Bei dem ersten, einem in einem Mischverfahren von Kupferstich und Radierung erstelltem Bild, handelt es sich um ein Profilportrait mit einem runden, illusorischen Schatten und mit einem Band verzierten

25 Zentralbibliothek Zürich, FA Lav. Ms. 504.243, 1, 1⁄4.

26 Gotthard J. Hansen, Die Sammlungen inländischen Alterthümer und anderen aus die baltischen Provinzen bezüglichen Gegenstände des estländischen Provinzial-Museums (Reval: Lindfors' Erben, 1875). Bekannt ist die Bleistiftzeichnung Lavaters von Lips, die um das Jahr 1780 entstand. - Einladung ins 18. Jahrhundert, hrsg. von Ernst-Peter Wieckenberg (München: C. H. Beck's Verlagsbuchhandlung, 1988), 51; eine Gravur von Lips, die Lavater darstellte: https://commons.wikimedia.org/wiki/ File:Johann_Caspar_Lavater._Line_engraving_by_J__H.__Lips_after_hi_Wellcome_V0003405ER.jpg 370 Gravuren am 27.07.2015); Lips fertigte von dem großen Werk, das Lavater abbildete, wenigsten der Menschenkenntnis und Menschenliebe; eine Auswahl mit 101 Abbildagmente zur Beforderung Siegrist (Stuttgart: Reclam, 1999), 378. 


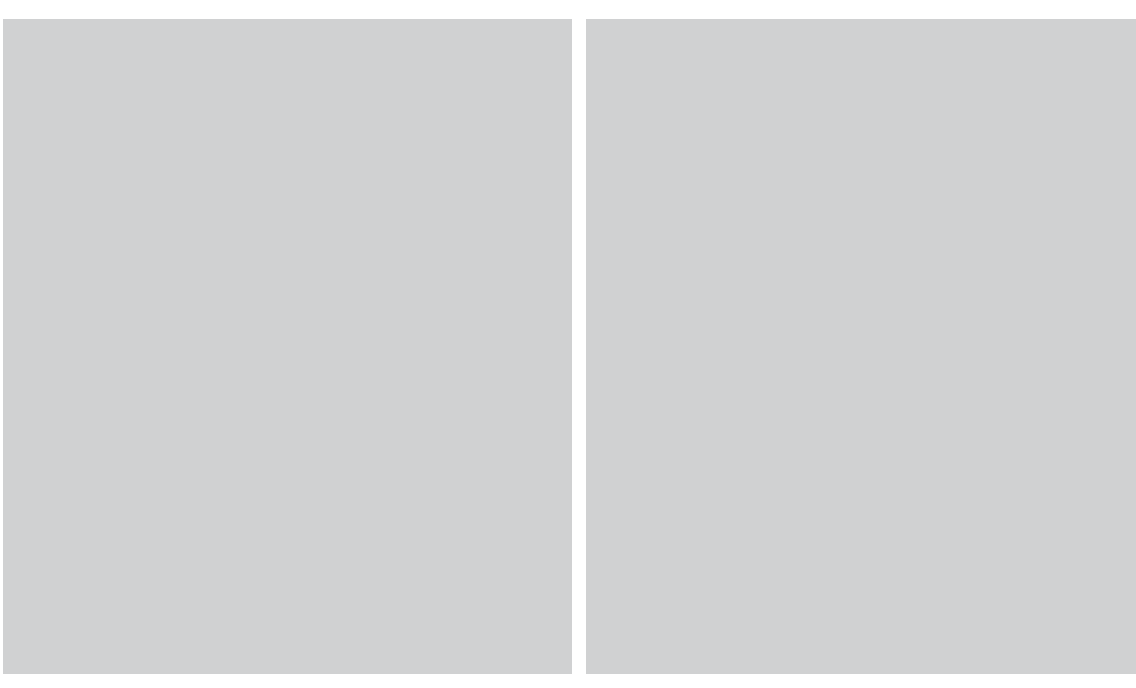

Abb. 3. Georg Friedrich Schmoll, Portrait Johann Caspar Lavaters, Kupferstich, Radierung.

Abb. 4. Georg Friedrich Schmoll, Portrait Johann Caspar Lavaters. Tallinner Stadtmuseum.

Rahmen, an dessen unten verborgener Seite auf eine Tafel der Name des Dargestellten geschrieben ist: IOHANN CASPAR LAUATER. Auf der unteren Seite des Papiers, die von der Druckplatte nicht erfasst wurde, ist aber folgender Text hinzugefügt: Verfertigt von Schmoll, und zu finden bey Steiner in Winterthur, was genauer auf Georg Friedrich Schmoll (gestorben 1785) als Stecher der Arbeit verweist. ${ }^{28}$ Schmolls Name findet sich auch unter dem Portrait Lavaters im Tallinner Stadtmuseum. Anders als im Falle der der Universität gehörenden Gravur ist diejenige des Stadtmuseums nicht in ihrer originalen Form erhalten, sondern die in der Technik des Kupferschnitts gefertigte Gravur wurde oval herausgeschnitten und auf ein Stück Papier geklebt, so wie auch die Tafel ausgeschnitten und auf dem Papier befestigt wurde. Diese verzeichnet den Namen des Abgebildeten, seinen Geburtsort und den Zeitpunkt, sowie Papierstreifen mit den Namen der Künstler - Schmoll (Zeichner) und J. E. Haid (der Gravierer Johann Elias Haid (1739-1809)) - sowie dem Datum Aug. 1777.

Die Portraits, welche der Universitätsbibliothek in Tartu und dem Tallinner Stadtmuseum gehören, zählen zu zwei von Hunderten nach

$\overline{28}$ Bibliothek der Universität Tartu, ÜR 458. der Zeichnung von Schmoll angefertigten grafischen Reproduktionen von Lavaters Profilportrait. Von dem mit den Namen Schmoll und Haid versehenen Portrait ist auch eine Variante im Schabkunst-Verfahren (Mezzotinto) bekannt, ebenfalls aus dem Jahr 1777.29

Das bildliche Format der Gravuren der Universitätsbibliothek und des Stadtmuseums, die Richtung von Lavaters Profil und die Unterschiede der Kleidung, verweisen auch auf die Tatsache, dass Schmoll Lavater mehrfach gemalt hatte oder dass sein Original von einem Meister, der unbekannt bleiben wollte, wiederholt wurde. Beide Varianten sind mit gleicher Wahrscheinlichkeit möglich. Auch Schmoll war Lavaters Mitarbeiter. Von ihm gezeichnete Portraits finden sich sowohl in Lavaters Kunstsammlung als auch unter den Buchillustrationen. ${ }^{30}$ Es besteht kein Zweifel, dass er über die Möglichkeit verfügte, Lavater zu begleiten und auf Papier zu verewigen. Lavaters Bekanntheit brachte eine große Nachfrage nach seinen Portraits mit sich. Deshalb wurden nicht nur seine besten, sondern auch die schlechteren Portraits unzählige Male kopiert. Was aber das dem Stadtmuseum gehörende Portrait betrifft und die oben beschriebene „Umformatierung” mit Hilfe von Schere und Leim, so war es Sitte in der Aufklärungszeit, so auch im Baltikum weit verbreitet, die Portraits bekannter Persönlichkeiten für sein eigenes Album persönlich zu gestalten. ${ }^{31}$

Wann das im Besitz der Universität befindliche Portrait nach Estland gelangte, ist nicht genau bekannt. Es erscheint als möglich, dass dies bereits vor der Wiedereröffnung der Universität im Jahr 1801 erfolgte, vielleicht durch den in Dorpat lebenden Major Oldekop, mit dem sich Lavater in einem ziemlich familiären Briefwechsel befand ${ }^{32}$ und der wie die Mehrzahl der Gelehrten der Zeit der Aufklärung eventuell neben den gelesenen oder gehörten Texten auch dessen Autor bildlich vor sich haben wollte. Von dem im Tallinner Stadtmuseum erhaltenen Portrait ist bekannt, dass dieses im Jahr 1963 vom Estnischen Kunstmuseum (estn.

29 Siehe: http://wellcomeimages.org/indexplus/image/V0003400.html (zuletzt besucht am 27.07.2015). 30 Althaus, ,Die Physiognomik ist ein neues Auge." Zum Porträt in der Sammlung Lavater, 209,
$215,231,232,235$. $215,231,232,235$.

Arbeiten im gleichen Format sind beispielsweise in der Kunstsammlung der Universitätsbibliothe in Tartu und in der Sammlung von Grafiken im Kunstmuseum Kadriorg (estn. Kadrior Kunstimuuseum) erhalten geblieben.

Ms. 522.185. Kopie des Schreibens im Besitz der Verfasserin. Wer Major Oldekop genau gewesen ist, konnte bis anhin nicht ermittelt werden. 
Eesti Kunstimuseum) übergeben wurde, ${ }^{33}$ wohin es seinerseits 1941 aus dem Bestand der liquidierten Estländischen Literärischen Gesellschaft (estn. Eestimaa Kirjanduse Ühing) gelangt war. ${ }^{34}$ Wann die Literärische Gesellschaft ein Portrait Lavaters für ihre Kunstsammlung erwarb, ist leider nicht bekannt. Gleichzeitig erscheint es als klar, dass es sich im Falle dieses gravierten Portraits nicht um den Schattenriss handeln konnte, den Lavater Burchard VII. gesandt hatte.

Über die Herkunft des vierten, heute dem Estnischen Kunstmuseum gehörenden Portrait Johann Caspar Lavaters (Abb. 5) ist bekannt, dass das Ölgemälde im Jahr 1953 im Rahmen einer Umstrukturierung der Sammlungen der Museen der Estnischen SSR ${ }^{35}$ dem Kunstmuseum des Staatlichen Ethnografiemuseum der Estnischen SSR (heute wieder Estnisches Nationalmuuseum, estn. Eesti Rahva Muuseum) übergeben wurde. ${ }^{36}$ In das letztere Museum gelangte das Gemälde im Jahr 1923 durch die vom Bildungsministerium erworbenen Kunstwerke aus der Sammlung von E. von Holst. ${ }^{37}$ Auf der Rückseite des Gemäldes befindet sich ein Textanhänger, demzufolge Lavaters Portrait auf den in Berlin und Hamburg erfolgenden Ausstellungen „Livland und Estland” ausgestellt war $^{38}$ als Anton Graffs Werk, ein im deutschen Sprachraum des Zeitalters der Aufklärung und im Baltikum hoch geschätzter Portraitmaler. ${ }^{39}$ Den richtigen Autor des Portraits, den Vertreter der Wiener Schule, doch mehrfach andernorts tätigen (Stuttgart, Ulm, Karlsruhe, Rom, Zürich usw.) August Friedrich Oelenhainz (1745-1804) fand in der ersten Hälfte der siebziger Jahre die estnische Kunsthistorikerin Juta

33 Übergabeakt Nr. 2 von Lavaters Portrait des Estnischen Kunstmuseums an das Tallinne Stadtmuseum vom 20.09.1963. - Archiv des Estnischen Kunstmuseums.

34 März 1941, Akt Nr. 65. - Archiv des Estnischen Kunstmuseu

36 Akt Nr. 432, 5. Januar 1953. - Archiv des Estnischen Kunstmuseums.

37 Das Gemälde,

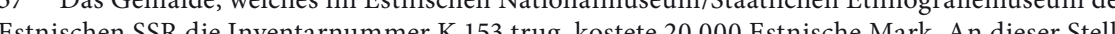
Estnischen SSR die Inventarnum 153 trug, kostete 20.000 Estnische Mark. An dieser Stelle danke ich

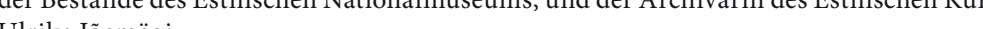

38 EKM M 2900 / VM 609, Information der Inventarkarte; Keevallik, „Märkmeid Anton Graff portreedest Eestis ja Latis", 335. Livland-Estland-Ausstellung: zur Einführung in die Arbeitsgebiet 39 über Gemälde des Künstlers, die im Baltikum gefunden wurden und Keevalliker Gemalde des Kunstlers, die im Baltikum gefunden wurden und zu finden sind, siehe Reevallik, „Markmeid Anton Graffi portreedest Eestis ja Latis, $330-340$. Zusatzlich sprechen fur heispie Kunstmuseums Kadriorg.

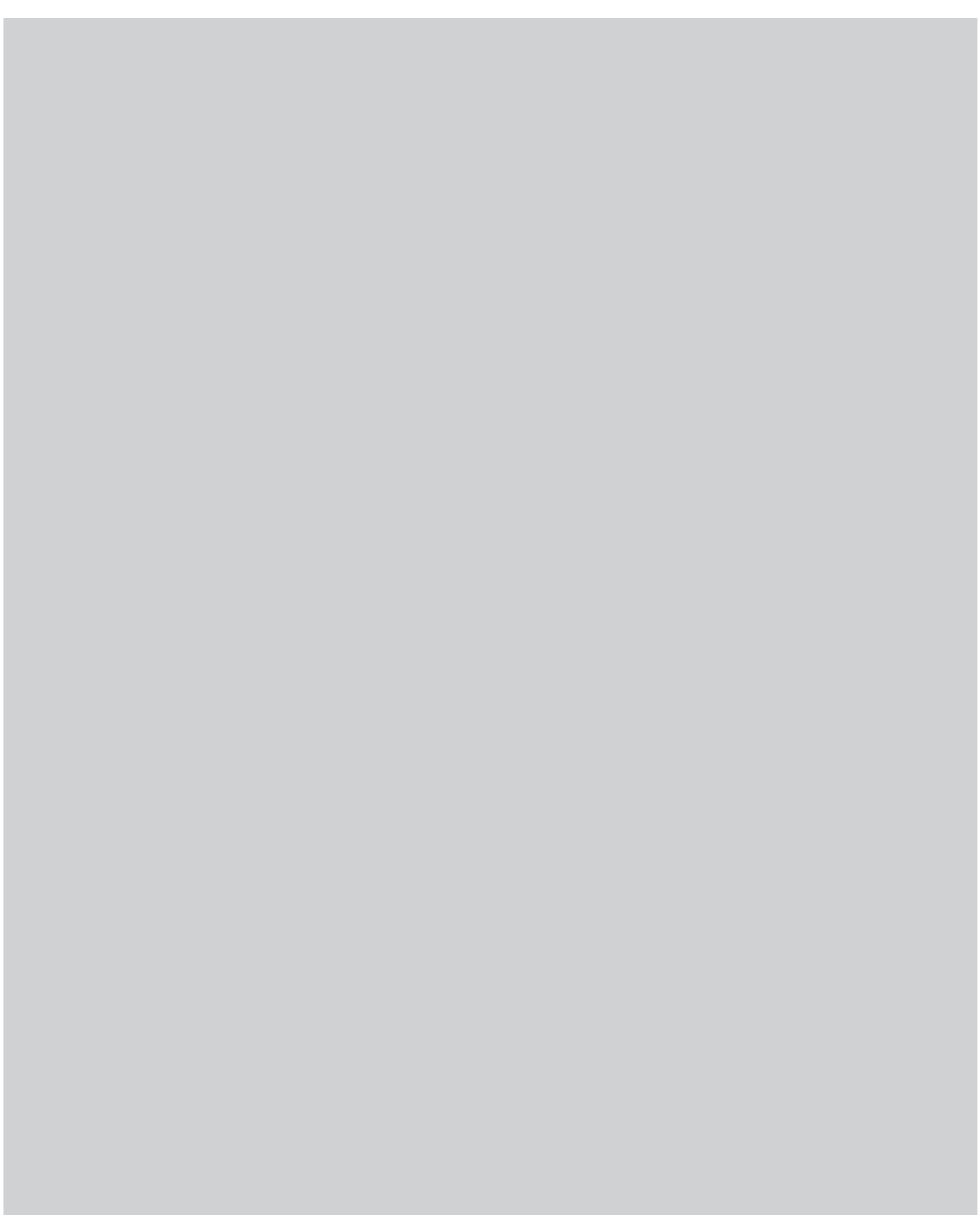

Abb. 5. Friedrich August Oelenhainz, Portrait Johann Caspar Lavaters, Öl auf Leinwand, etwa 1788-1791. Estnisches Kunstmuseum, Kunstmuseum Kadriorg.

Keevallik. Hinter der früheren falschen Zuschreibung stand, so vermutete Juta Keevallik, sowohl die Ähnlichkeit der Maltechnik von Oelenhainz mit Graffs späterer Schaffensperiode als auch Graffs persönliche Bekanntschaft mit Lavater. Die beiden Männer standen nicht 
nur miteinander im Schriftverkehr ${ }^{40}$ sondern sie haben sich auch mehrfach persönlich getroffen. Graff war der Schwiegersohn von Lavaters Freund Johann Georg Sulzer (1720-1779); er fertigte von Lavater auch einige Portraitzeichnungen an. ${ }^{41}$ Lavater hat die Arbeiten Graffs wiederholt für seine physiognomischen Studien benutzt, zum Beispiel das Portrait von Graffs Gattin. ${ }^{42}$

Das sich im Kunstmuseum befindliche Werk von Oelenhainz mit der Bezeichnung "Johann Caspar Lavaters Portrait" ist außerhalb Estlands wenig bekannt. Nach Auffassung Juta Keevalliks könnte das Portrait aus den Jahren 1790 bis 1795 stammen, was gleichzeitig auch der Höhepunkt des Schaffens des Künstlers war. Mit Hinweis auf das im Zürcher Pestalozzi-Museum erhaltenes Portrait Lavaters von Oelenhainz aus dem Jahre 1788 (das als Stich von C. H. Pfeiffer verbreitet ist), welches dem Tallinner Gemälde sehr ähnelt, vermutet Keevallik, dass das Werk des Estnischen Kunstmuseums aus den Jahren 1790-1791 nach dessen Vorbild von Oelenhainz selbst als Kopie angefertigt worden sei. ${ }^{43}$

Zugleich besteht auch eine zweite Möglichkeit. Gestützt auf die von Professor Leopold Oelenhainz im Jahr 1907 veröffentlichten Monografie, kann vermutet werden, dass tatsächlich das Zürcher (in dem früheren Pestalozzistübchen hängende) Gemälde für das nach Tallinn gelangte Portrait „eine verloren gegangene” oder „verschollene"44 Vorarbeit von Lavaters Portrait ist. Wenn dem tatsächlich so ist, dann könnte das Tallinner Portrait älter sein, da es unmittelbar nach der 1788 abgeschlossenen Vorarbeit gemalt wurde. ${ }^{45}$ Dass es sich tatsächlich um zwei verschiedene Gemälde handelt, bestätigt einerseits die Tatsache, dass laut Leopold Oelenhainz beim Zürcher Portrait die Oberflächenbearbeitung nicht beendet worden war und andererseits der Umstand, dass im Vergleich zum Tallinner Werk das Zürcher Gemälde ein wenig kleiner ist: 75x60 cm gegenüber 71x54 cm. ${ }^{46}$ Die Tatsache, dass Oelenhainz

40 Johann Caspar Lavater Ausgewählte Werke. Ergänzungsband: Verzeichnisse der Korrespondenz und des Nachlasses in der Zentralbibliothek Zürich, 76, 182

1 Keevallik, „Märkmeid Anton Graffi portreedest Eestis ja Lätis”, 337-338.

42 Lavater, Physiognomische Fragmente, III, 302.

43 Früher wurde Lavaters Portrait aus dem Jahre 1791 Wilhelm Wartmann zugeschrieben, „Diogg und Lavater", Zeitschrift für schweizerische Archäologie und Kunstgeschichte, 3 (1941), 177.

4 Leopold Oelenhainz, Friedrich Oelenhainz. Ein Bildnismaler des 18. Jahrhunderts. Sein Leben und seine Werke (Leipzig: E. A. Seemann, 1907), 22.

45 Das Portrait gehörte am Anfang des 20. Jahrhunderts dem Direktor des Pestalozzimuseums,

46 Oelenhainz, Friedrich Oelenhainz, 49 .
Portrait von Lavater zu seiner Zeit als gelungen angesehen wurde, wird dadurch bestätigt, dass eine weitere Kopie dessen (mit den Maßen 52,2x41 $\mathrm{cm}$ ) im Frankfurter Goethe-Haus hängt. ${ }^{47}$

Professor Oelenhainz hielt „Lavaters Portrait” für gelungen vor allem wegen dessen großer Ähnlichkeit mit dem Abgebildeten: „Die feinen, festen Züge Lavaters, dieses seltenen Mannes, das klarerfassende Auge, die feine schöne Hand haben in Oelenhainz den besten Darsteller gefunden. /---/ Ein enganliegendes schwarzes Sammetkäppchen faßt die seitlich etwas grauen Haare. Hemdkragen über die schwarze Sammetweste und dem graubraunen Rock vorn breit aufgeschlagen. Farbengebung nich lebhaft. Hand besonders schön gemalt. Sehr ausdrucksvolles Bild." ${ }^{\prime 4}$

Die Genauigkeit und treffliche Sicherheit erklärt teilweise die spätere grafische Reproduzierung des Lavater-Portraits von Oelenhainz durch Carl Hermann Pfeiffer (1769-1829). ${ }^{49}$ Die zweite und die dritte Ursache für die Wiederholung des gelungenen Portraits könnte aber gewesen sein, dass an der Verbreitung des Bildes sowohl der Künstler als auch sein Modell aus verschiedenen Motiven interessiert waren. Friedrich August Oelenhainz, der im Atelier seines Onkels, eines Malers, die künstlerische Ausbildung begonnen hatte, diese beim Hofmaler Württembergs, Johann Wilhelm Beyer, fortsetzte, dann an der Kunstakademie in Wien Malere studiert hatte und zu einem der Lieblingsportraitmaler des österreichischen Kaiserhofs aufgestiegen war, war daran interessiert, dass sich seine Werke und damit auch sein Ruhm als Künstler verbreiteten. Auch der Zürcher Pfarrer, Schriftsteller, Dichter und Physiognom Johann Caspar Lavater hatte ein Interesse, dass seine Berühmtheit nicht nur durch seine Werke und Briefe, sondern auch durch die bildliche Vermittlung wachse In der Zeit der Aufklärung begann man dem mit den Augen Sichtbaren vermehrt Bedeutung zuzuschreiben. Der „Leserevolution” sekundierte eine „Bildexplosion”, und aus Bildern wurde so eine Wissenschaft. In der Pädagogik sekundierte man dazu mit der Hervorhebung des Visuellen, Bildlichen. ${ }^{50}$ Auf die Möglichkeit, dass der Auftraggeber des Tallinner

47 Frankfurter Goethe-Haus, Bildnummer: ART27004

48 Oelenhainz, Friedrich Oelenhainz, 22, 49.

49 Siehe: http://prometheus.uni-koeln.de/pandora/de/image/show/heidicongs-63e67043bfd437e 7b36c75af7d2ee0038dba333e (zuletzt besucht am 22.09.2015). Zusätzlich zur beendeten Gravur is in der Zentralbibliothek Zürich auch ein Probedruck des Portraits erhalten, veröffentlicht in: Ursula Caflisch-Schnetzler, „Pädagogik und Kommunikation - Zurich und "Kulturaustausch.

50 Althaus, Die Physiognomik ist ein neues Auge," Zum Porträt in der Sammlung Lavater, 33-84. 
Portraits Lavater selbst oder eine deutschbaltische Persönlichkeit gewesen sein könnte, hat auch Juta Keevallik hingewiesen - Lavater war in Est-, Liv- und Kurland mit mehreren Personen in Kontakt gewesen. ${ }^{51}$ Wann Lavaters Portrait in die Hände des ersten bekannten Eigentümers, in den Besitz der Familie von Dr. Holst, gelangte, konnte bedauerlicherweise noch nicht ermittelt werden. Möglicherweise erfolgte dies bereits Ende des 18. Jahrhunderts oder zu Beginn des 19. Jahrhunderts, als das Geschlecht der Holsts, das dem Baltikum mehrere bekannte Literaten geschenkt hat, bereits (und vielleicht in Tartu) ansässig war. ${ }^{52}$

\section{LAVATER ÜBER DIE PORTR AITKUNST}

Die Portraitkunst war für Lavater von besonderer Bedeutung. Bei der Untersuchung des menschlichen Gesichts versuchte er nicht nur, die Menschen zu verstehen und die zwischenmenschlichen Beziehungen zu erleichtern, mit anderen Worten, die Nächstenliebe zu befördern, sondern auch, und dies ist für ihn als Theologen besonders interessant, das Angesicht Gottes zu finden. Lavater glaubte nämlich, dass Gott den Menschen nach seinem Antlitz geschaffen hatte (1 Mo 1.27) und er folgerte daraus, dass das Gesicht eines jeden Menschen auf eine besondere Weise etwas über das Gesicht Gottes aussagt. ${ }^{53}$

Dieser Ausgangspunkt erklärt Lavaters besondere Ansprüche an die Portraitisten sowie die heftige Kritik an den Portraits. Gut bekannt ist denn Lavaters Kritik an dem ihn portraitierenden Wilhelm Tischbein, ${ }^{54}$ aber auch an Künstlern, die zu seinen Mitarbeitern zählten, auf deren Arbeit sich seine eigene Arbeit und seine Bekanntheit schließlich stützten. ${ }^{55}$

In den „Physiognomischen Fragmenten” widmete Lavater der Portraitkunst ein eigenes Kapitel, aus dem einige fragmentarische Auszüge folgen: „Die natürlichste, menschlichste, edelste, nützlichste

51 Keevallik, „Märkmeid Anton Graffi portreedest Eestis ja Lätis”, 338.

52 Baltisches Biographisches Lexikon (digital: http://www.bbl-digital.de/eintrag/Holst\%2C-v./ zuletzt besucht am 15.11.2015)

53 Ausführlicher siehe beispielsweise Ueli Greminger, Johann Caspar Lavater. Berühmt, berüchtigt - neu entdeckt (Zürich: Theologische Verlag Zürich, 2012), 46-55; Siegrist, „Nachwort”, 379-380. 54 Ibidem, 206.

55 Die wichtigsten Mitarbeiter Lavaters waren Johann Heinrich Lips (1758-1817), der wenigstens 370 Gravuren anfertigte, und Johann Rudolf Schellenberg (1740-1806), von dem 91 Gravuren stammen; (801). Siegiste 1801). Siegrist, „Nachwort”, 378
Kunst, - und die schwerste, so leicht sie scheint, so leicht sie seyn sollte die Porträtmahlerey. /---/ Wenn es wahr ist, was Goethe irgendwo sag - und mich dünkt, Wahrers läßt sich nichts sagen - „daß des Menschen Gegenwart, daß sein Gesicht, seine Physiognomie, der beste Text zu allem ist, was immer über ihn gesagt und commentirt werden kann" wie wichtig wird die Porträtmahlerey! /---/ Wie heilig und ehrwürdig also sollte dem Mahler das gemeinste Menschenangesicht seyn! /---/ wer nicht die Seele im Gesichte sieht, kann sie nicht mahlen - und wer diese nicht mahlen kann, ist kein Porträtmahler. /---/ Jedes vollkommne Porträt ist ein wichtiges Gemählde, weil es uns eine menschliche Seele von einem persönlichem Charakter zu erkennen giebt; wir sehen in demselben ein Wesen, in welchem Verstand, Neigungen, Gesinnungen, Leidenschaften, gute und schlimme Eigenschaften des Geistes und des Herzens auf eine ihm eigne und besondre Art gemischt sind. Dieses sehen wir sogar im Porträt meistentheils besser, als in der Natur selbst; weil hier nichts beständig, sondern schnell vorübergehend und abwechselnd ist."

Im weiteren folgt Lavaters Aufruf zur Wachsamkeit bei der Betrachtung von Menschen in der Abbildung und bezüglich der Bewertung eines Portraits: „So viel ich Porträtmahler gesehen, so viele Werke von Porträtmahlern, so oft bemerkt' ich Mangel an philosophischer, das ist, richtiger, deutlicher, und zugleich allgemeiner Kenntniß - des Menschen. /---/ Der Insektenmahler, der keine genaue Insektenkenntniß hat, nicht den Bau, das Allgemeine, das Besondere, das Eigenthümliche jedes Insektes kennt, wird, wenn er sonst überhaupt auch noch so ein guter Copist ist - unfehlbar schlecht Insekten mahlen. Der Porträtmahler könne noch so genau kopieren - (eine Sache, die jedoch weit seltener ist, als selber große Kenner der Zeichnung denken mögen -) er wird schlechte Porträte mahlen; wenn er nicht die genaueste Kenntniß hat von dem Bau, der Proportion, dem Zusammenhange, der Gegeneinanderwürkung der gröbern und feinern Theile des menschlichen Körpers, in so fern sie auf die Oberfläche einen merkbaren Einfluß haben; wenn er nicht den Bau jedes einzelnen Gliedes und Gesichtstheiles aufs genaueste ergründet hat; etwas, das ich noch schlechterdings_an keinem einzigen mir bekannt gewordenen Porträtmahler gefunden habe." ${ }^{\prime 56}$

56 Lavater, Physiognomische Fragmente, II, 78-89. 
Nach einer solch scharfen Stellungnahme - die meisten gemalten Portrait sind nicht vollkommen, da das genaue Gesichtsstudium fehlt - setzt Lavater fort: „Was hilft, sag' ich, alle Kenntniß der größern Proportionen, wenn die Kenntniß der feinern Züge, die eben so wahr, so allgemein, so bestimmt, und nicht weniger bedeutend sind, als die größern - wenn diese fehlt? /---/ Man durchgehe dreyßig, vierzig Bände der trefflichsten Porträte von den größten Meistern, und untersuche - (ich hab' untersucht, und darf also kühn sprechen) wie gesagt, nur z. E. den Mund. Studiere vorher an neugebornen Kindern, Knaben, Jünglingen, Männern, Greisen, Jungfrauen, Frauen, Matronen - das Allgemeine des Mundes, und wenn man's gefunden hat, so vergleiche man - und man wird sehen, daß den meisten, daß beynah allen Mahlern die Theorie des Allgemeinen des Mundes fehlet, und daß es sehr selten geschieht, wenn's geschieht, bloß zufälligerweise zu geschehen scheint, daß ein Meister dieß Allgemeine richtig gefaßt hat? /---/ Und wie's in Ansehung des Mundes ist, so in Ansehung der Augen, der Augenbraunen, der Nase und jedes Gliedes oder Gesichtstheils..." ${ }^{57}$

$\mathrm{Zu}$ jenen und anderen Bemerkungen an die Adresse der Portraitisten die sich nicht genug bemühten, sich in die von Gott geschaffene Natur ausreichend zu vertiefen, sondern den Menschen nach eigenem Ermessen abbilden, sei dies wegen fehlendem Talent oder wegen der Verfolgung eigener künstlerischer Ziele, folgt in den „Physiologischen Fragmenten” konkrete Kritik am Beispiel von insgesamt hundert Einzelfällen. Auch bezüglich Johann Wolfgang von Goethes Portrait (Abb. 6), welches Lavater im Allgemeinen für gelungen hält, vermag er nicht ohne Kritik zu loben: „Steinern nach Stein gearbeitet; aber äußerst charakteristisch für den Physiognomiker. Immer Larve eines großen Mannes, der das Creditif seiner Vollmacht auf die Menschheit zu würken auf seinem Gesichte hat; - sogar auf der harten Larve seines Gesichtes. Auch ohne das blitzende Auge; auch ohne die geistlebendige Lippe, auch ohne die blaßgelblichte Farbe - auch ohne den Anblick der leichten, bestimmten, und alltreffenden, allanziehenden, und sanft wegdrängenden Bewegung - ohn' alles das ... welche Einfachheit und Großheit in diesem Gesichte! In der Stirne bis zur Augenbraune heller, richtiger, schneller Verstand. Sehr zwar wird der Eindruck dieser Stirne wieder verwischt durch den zu gedehnten und gewölbten Vorbug, von der Augenbraune an bis an die Wurzel der

57 Lavater, Physiognomische Fragmente, II, 78-89.

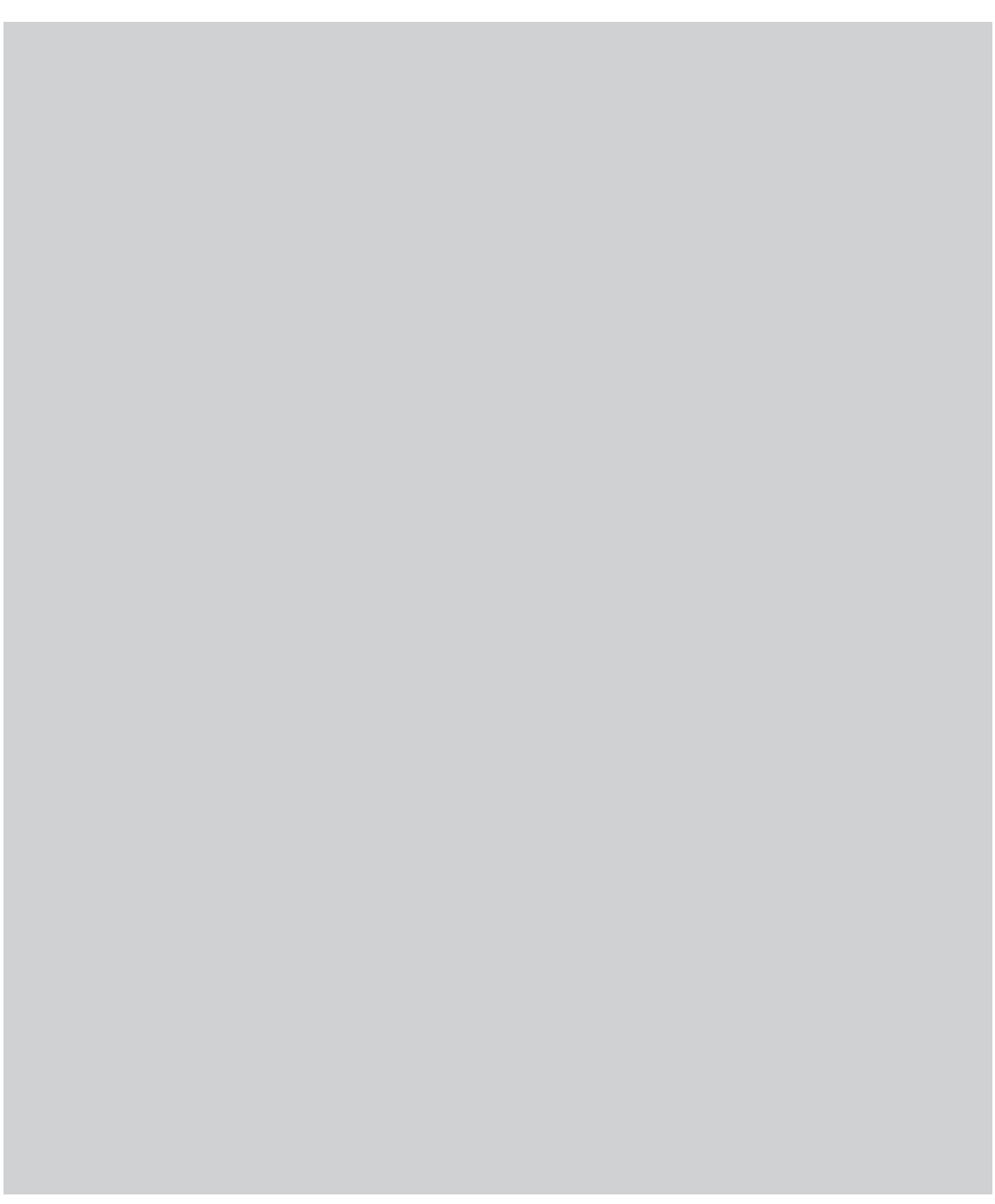

Abb. 6. [Johann Wolfgang von Goethe, Portrait], 3. Versuch, 218. Foto: Akademische Bibliothek der Universität Tallinn.

Nase. Das Auge hier hat bloß noch im obern Augenliede Spuren des kraftvollen Genius. Der Augapfel selber ist in aller Betrachtung unerträglich. /---/ Die Unterlippe ist zu rund abgeschliffen, und kontrastirt dadurch sehr mit der viel delikatern Oberlippe. Das Kinn trefflich; besonders der Kinnball. Nur um ein Haar zu kleinlich. Der mächtige Zug von Aug und 
Mund herab unwahr; voll Ernst und Stolz." ${ }^{58}$ (Ähnliche Analysen von Portraits finden sich zahlreich in den „Physiognomischen Fragmenten”.)

Hierbei ist aber nötig zusetzen, dass Lavaters Physiognomik nicht nur die Künstler, sondern zehntausend, ja hunderttausend Leser beeinflusste, die nach der Lektüre der „Physiognomischen Fragmente” nicht nur die Natur des Menschen, sondern auch die Portraitkunst mit einem neuen Blick zu betrachten oder zu bewerten begannen.

\section{ZUM SCHLUSS}

Der Einfluss von Lavater im Kulturraum des Baltikums, in der Kulturund enger in der Kunstgeschichte ist bemerkenswert sowohl wegen seiner hiesigen Kontakte, wegen seiner "Physiognomische Fragmente” als auch angesichts der Existenz seiner Portraits. Lavaters Untersuchungen des menschlichen Gesichts und der Bemerkungen zur Portraitkunst haben gewiss auch die Rezeption der schnellanwachsenden Anzahl von Silhouettenportraits in örtlichen Haushalten und Sammlungen beeinflusst. Die Kenntnis von Lavater hilft sicherlich den Versuchen jener Kunstwissenschaftler, die probieren, das Visuelle der Zeit der Aufklärung, darunter die Portraitkunst, im eigenen Rahmen der Aufklärungszeit zu betrachten und dabei den Einfluss der Arbeiten Lavaters auf seine Zeit und die Kunst wahrzunehmen. ${ }^{59}$

Lavaters eigene Kenntnisse, seine physiognomischen Studien, können für die Betrachtung der ihn abbildenden Portraits (aber auch die seiner Zeitgenossen) ein Schlüssel sein. Ein Schlüssel, welchen die Gedächtnisinstitutionen in Estland, darunter das Estnisches Kunstmuseum - Kunstmuseum Kadriorg (im Schloss Kadriorg, estn. Eesti Kunstimuuseum - Kadrioru kunstimuuseum), welches das bemerkenswerte, von August Friedrich Oelenhainz gemalte Portrait Lavaters in der ständigen Ausstellung hält, Tag für Tag brauchen.

58 Lavater, Physiognomische Fragmente, III, 218

59 Ein entsprechender Versuch wurde im Mikkel-Museum des Estnischen Kunstmuseums in Kadriorg mit der Ausstellung „, Johann Caspar Lavaters Gesichterbuch gemacht. Der Blick der Aufklärungszeit Kreem). Denschen und die Kunst (24.10.2015-27.03.2016, Kuratoren Anu Allikvee und Tiina-Mall Kreem). Die Ausstellung begleiten folgende Veröffentlichungen: Tunne inimest! Johann Caspar Lavater, 2015): vohann Caspar Lavateri nä̈ll Kren (Tallinn. Tallinna Ulikooli Akedeeniline Raamatukogu, Allikvee und Tiina-Mall Kreem (Tallinn: Eesti Kunstimuuseum, 2015).
Tiina-Mall Kreem: Johann Caspar Lavater. On His Influence, Some Portraits and Reflections on The Art of Portraiture

Keywords: EnLightenMENT-era PORTRAiture; PHYSIOGNOMY; Baltic, GERMAN, SWISS CULTURAL CONTACTS

\section{SUMMARY:}

The article focuses on Johann Caspar Lavater (1741-1801), the Enlightenment-era thinker, pastor and writer, art collector and physiognomist, whose work and activities affected thinking from Zurich to America and Russia, including the Baltic countries. Of Lavater's Estonian acquaintances, Johann Burchard VII, the Tallinn Town Council pharmacist, is the one that primarily emerges from the article. The famous Swiss maintained a correspondence with the latter for over ten years, and in 1792, gifted him a miniature portrait of himself (now in the Estonian History Museum).

In addition to the miniature portrait after Johann Heinrich Lips (?), there were two graphic portraits of Lavater in Estonia that were associated with Georg Friedrich Schmoll (Tallinn City Museum, University of Tartu Library) as well as a masterful oil portrait by August Friedrich Olenhainz (Art Museum of Estonia's Kadriorg Art Museum). The article examines all of these against the background of Lavater's successful book of the day "Physiognomische Fragmente zur Beförderung der Menschenkenntnis und Menschenliebe" ("Physiognomic Fragments for Furthering the Knowledge and Love of Man", 1775-1778) and in regard to Lavater's discussions about people and the art of portraiture.

An attempt is made thereby to see Enlightenment-era portrait art through the eyes of Enlightenment-era people - Lavater and his audience. While the author of the article is convinced of the impact of Lavater's physiognomic research on the portraiture of the day (on the artists, clients, viewers) and also more indirectly on the history of art, she emphasis that, for Lavater, portrait art was primarily a tool for his physiognomic research and even if Lavater's teachings lost their popularity after his death and were relegated to the periphery of science, Lavater should not be excluded from the history of art and culture in the Baltic countries. 
CV:

Dr. Tiina-Mall Kreem (b. 1971) has worked as the Curator of the Estonian and Foreign Sculpture Collection at the Art Museum of Estonia since 1993. In addition to the history of sculpture, her main fields of research include $19^{\text {th }}$ century sacral architecture and art, as well as Estonian historical pictures from the $19^{\text {th }}$ to $21^{\text {st }}$ century. 